\title{
Voxel-based lesion mapping of meningioma: a comprehensive lesion location mapping of 260 lesions
}

\author{
Ryuichi Hirayama, MD,1 Manabu Kinoshita, MD, PhD, ${ }^{1,2}$ Hideyuki Arita, MD, PhD, ${ }^{1}$ \\ Naoki Kagawa, MD, PhD, ${ }^{1}$ Haruhiko Kishima, MD, PhD, ${ }^{1}$ Naoya Hashimoto, MD, PhD, ${ }^{1}$ \\ Yasunori Fujimoto, MD, PhD, ${ }^{1}$ and Toshiki Yoshimine, MD, PhD' \\ 'Department of Neurosurgery, Osaka University Graduate School of Medicine, Suita, Osaka; and 'Department of Neurosurgery, \\ Osaka International Cancer Institute, Osaka, Japan
}

OBJECTIVE In the present study the authors aimed to determine preferred locations of meningiomas by avoiding descriptive analysis and instead using voxel-based lesion mapping and $3 \mathrm{D}$ image-rendering techniques.

METHODS Magnetic resonance images obtained in 248 treatment-naive meningioma patients with 260 lesions were retrospectively and consecutively collected. All images were registered to a 1-mm isotropic, high-resolution, T1-weighted brain atlas provided by the Montreal Neurological Institute (the MNI152), and a lesion frequency map was created, followed by $3 \mathrm{D}$ volume rendering to visualize the preferred locations of meningiomas in $3 \mathrm{D}$.

RESULTS The 3D lesion frequency map clearly showed that skull base structures such as parasellar, sphenoid wing, and petroclival regions were commonly affected by the tumor. The middle one-third of the superior sagittal sinus was most commonly affected in parasagittal tumors. Substantial lesion accumulation was observed around the leptomeninges covering the central sulcus and the sylvian fissure, with very few lesions observed at the frontal, parietal, and occipital convexities.

CONCLUSIONS Using an objective visualization method, meningiomas were shown to be located around the middle third of the superior sagittal sinus, the perisylvian convexity, and the skull base. These observations, which are in line with previous descriptive analyses, justify further use of voxel-based lesion mapping techniques to help understand the biological nature of this disease.

https://thejns.org/doi/abs/10.3171/2017.3.JNS17169

KEY WORDS meningioma; lesion location preference; voxel-based lesion mapping; MNI152 coordinates; 3D visualization; oncology

$\mathrm{M}$ ENINGIOMAS are one of the most common intracranial neoplasms. They constitute approximately one-third of primary CNS tumors, and in most cases present a benign clinical course. ${ }^{3,8,9}$ As the number of neuroimaging examinations being performed increases worldwide, more of these tumors are being discovered incidentally or in association with a very minor neurological deficit in the patient. ${ }^{13,14}$ Surgical risks associated with tumor removal impact patient morbidity, and pose difficult decisions for both clinicians and patients. Meningiomas are considered to derive from arachnoidal cap cells of the leptomeninges. ${ }^{3}$ Findings have recently been published that meningiomas can be categorized into several subgroups according to their different genetic mutations. , $^{2,416}$ More interestingly, it was suggested that the genetic alterations seen in these tumors correlate with the locations where they arise within the cranium. ${ }^{4}$

Although typical locations of meningiomas have been documented since the 1920s during the era of Harvey Cushing, ${ }^{6,7}$ previous studies have relied on descriptive

ABBREVIATIONS FSL-FLIRT = FSL Linear Image Registration Tool; JBTR = Japan Brain Tumor Registry; Mango = Multimage Analysis GUI; MNI152 = Montreal Neurological Institute brain atlas; NF2 = neurofibromatosis Type 2; NIfTI = Neuroimaging Informatics Technology Initiative; UCSF = University of California, San Francisco. SUBMITTED January 18, 2017. ACCEPTED March 10, 2017.

INCLUDE WHEN CITING Published online September 1, 2017; DOI: 10.3171/2017.3.JNS17169. 
TABLE 1. Demographic data in 248 patients with meningioma

\begin{tabular}{lccc}
\hline \multicolumn{1}{c}{ Variable } & All & Op Case & Nonop Case \\
\hline No. of cases (\%) & 248 & $114(46.0)$ & $134(54.0)$ \\
\hline Mean age in yrs (range) & $61(22-90)$ & $58(22-82)$ & $64(38-90)$ \\
\hline Sex ratio (F:M) & $190: 58$ & $80: 34$ & $110: 24$ \\
\hline $\begin{array}{c}\text { Lesion laterality } \\
\text { (rt:lt:mid:multi) }\end{array}$ & $91: 117: 27: 13$ & $40: 50: 19: 5$ & $51: 67: 8: 8$ \\
\hline
\end{tabular}

Mid = middle; multi $=$ multiple .

analysis of locations. This type of analysis makes it difficult to gain a perspective on the entire spatial landscape of meningioma occurrence. Therefore, the present study aims to overcome this problem and to determine the preferred locations of these tumors in a global manner by the use of voxel-based lesion mapping and 3D image-rendering techniques.

\section{Methods}

\section{Patient Population}

Pretreatment MR images obtained in patients with meningioma were consecutively collected. All patients were referred to our institution between 2000 and 2013. In total, 260 lesions among 248 patients were included for analysis. The use of clinical data was approved for research purposes by the local institutional review board. Detailed demographic information about the patients is listed in Table 1. All the patients were evaluated by board-certified diagnostic radiologists according to a definition of meningiomas as extraaxial masses with broad-based attachment along the dura mater or the choroid plexus in the ventricles, and which are homogeneously and markedly enhanced with contrast medium, as described in a previous study. ${ }^{22}$ Two cases had a clinical history of radiation therapy, and 6 surgically treated cases were diagnosed as atypical meningio- mas. No patient with neurofibromatosis was included for analysis.

\section{Magnetic Resonance Imaging}

The MRI studies were performed at either $1.5 \mathrm{~T}$ (Signa Genesis/Excite, GE Healthcare; or Magnetom Vision Plus, Siemens) or 3 T (Signa HDxt, GE Healthcare; or Achieva/Ingenia, Philips Healthcare). Voxel sizes were as follows (in the order of minimum, median, maximum, and mean) $-x$-dimension: $0.35,0.47,1.4$, and $0.63 \mathrm{~mm}$; $y$ dimension: $0.35,0.47,5.9$, and $0.66 \mathrm{~mm}$; and z-dimension: $0.43,1,7.2$, and $2.8 \mathrm{~mm}$.

\section{Definition of Tumor Location}

Tumor location was determined using MR images. In cases in which the tumor was too large to determine its exact origin, ${ }^{9}$ it was defined as the most widely attached portion of the tumor to the dura mater. The location of the tumor was defined according to the classification system used in the Japan Brain Tumor Registry ${ }^{5}$ (Table 2). The tumor location distribution data were compared with those obtained from the Japan Brain Tumor Registry and those from a previous publication by the University of California, San Francisco (UCSF). ${ }^{11}$ Chi-square tests were used for group comparisons and a $p$ value of less than 0.05 was considered statistically significant. Statistical analysis was performed using Prism 5 (GraphPad Software, Inc.).

\section{Voxel-Based Lesion Mapping and 3D Rendering of Meningioma}

Voxel-based lesion mapping was performed by modifying methodologies used in previous reports. ${ }^{12,19}$ All Digital Imaging and Communications in Medicine (DICOM) format images were first converted to the Neuroimaging Informatics Technology Initiative (NIfTI) format

TABLE 2. Distribution of meningioma locations in the present series compared with reports in the literature

\begin{tabular}{|c|c|c|c|c|}
\hline \multirow[b]{2}{*}{ Location } & \multirow{2}{*}{$\begin{array}{c}\% \text { (no.) in } \\
\text { Present } \\
\text { Series }\end{array}$} & \multicolumn{3}{|c|}{ Reports in the Literature } \\
\hline & & $\begin{array}{l}\% \text { (no.) in JBTR, } \\
2001-2004\end{array}$ & $\begin{array}{c}\% \text { (no.) in UCSF Series, } \\
2000-2007\end{array}$ & $\begin{array}{l}\text { Original Classification } \\
\text { in UCSF Report }\end{array}$ \\
\hline Convexity & $25(65)$ & $23.9(807)$ & $24.9(94)$ & Convexity, extensive convexity \\
\hline Parasagittal & $16.9(44)$ & $10.7(361)$ & Not reported & \\
\hline Cerebellopontine angle & $9.2(24)$ & $7.7(261)$ & $7.1(27)$ & Cerebellopontine angle \\
\hline Falx & $8.1(21)$ & $9.2(309)$ & $16.1(61)$ & Falx \\
\hline Sphenoid wing & $8.1(21)$ & $10.3(347)$ & $10.3(39)$ & Sphenoid wing \\
\hline Tentorium & $7.3(19)$ & $5.5(187)$ & $0.5(2)$ & Tentorium \\
\hline Parasellar/cavernous sinus & $11.2(29)$ & $11.9(401)$ & $16.4(62)$ & Cavernous sinus, clinoid, parasellar, planum, tuberculum \\
\hline Middle fossa & $1.2(3)$ & $2.8(93)$ & $2.1(8)$ & Middle fossa \\
\hline Olfactory groove & $3.8(10)$ & $4.4(147)$ & $3.7(14)$ & Olfactory groove \\
\hline Cerebellar convexity & $3.8(10)$ & $1.6(55)$ & $5.6(21)$ & Posterior fossa \\
\hline Clivus/petroclival & $3.5(9)$ & $5.8(197)$ & $2.4(9)$ & Clivus, petroclival, petrous \\
\hline Intraventricular & $1.2(3)$ & $1.8(62)$ & $1.9(7)$ & Intraventricular \\
\hline Foramen magnum & $0.4(1)$ & $1.1(37)$ & $3.2(12)$ & Foramen magnum \\
\hline Other & $0.4(1)$ & $3.2(107)$ & $5.8(22)$ & Multifocal, orbit, jugular foramen \\
\hline Total & $100(260)$ & $100(3371)$ & $100(378)$ & \\
\hline
\end{tabular}




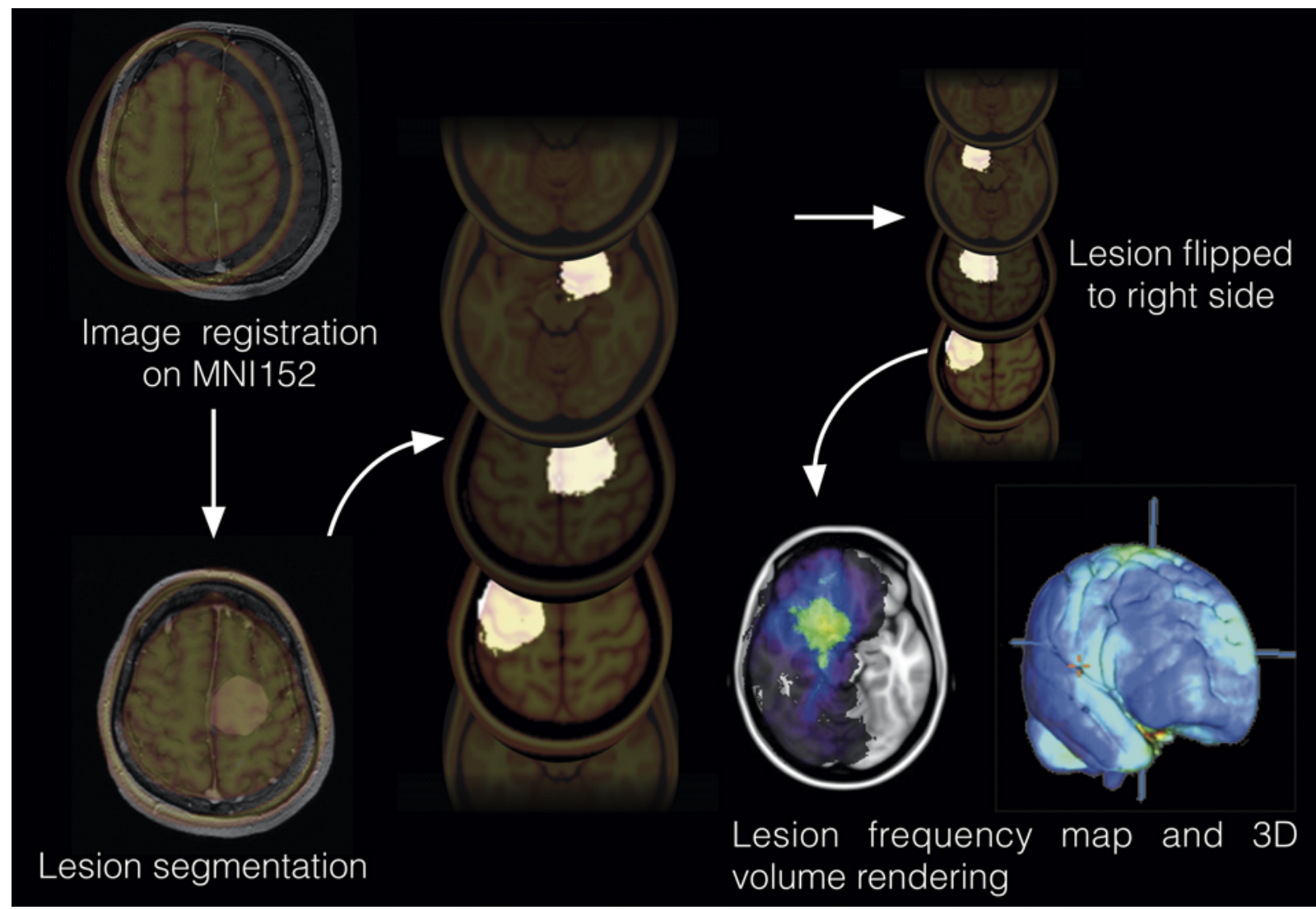

FIG. 1. Workflow for voxel-based lesion mapping. The NIfTI data were registered to a 1-mm isotropic, high-resolution, T1-weighted brain atlas provided by the MNI (the MNI152). The Gd-enhancing lesions were semiautomatically segmented and flipped so that all lesions were concentrated onto the right side of the brain. All lesions were then summed and a heat map for the frequency of lesion occurrence was reconstructed, followed by $3 \mathrm{D}$ rendering of the frequency map.

by using MRIconvert (University of Oregon Lewis Center for Neuroimaging: http://lcni.uoregon.edu/ jolinda/ MRIConvert/). The NIfTI data were registered to a 1-mm isotropic, high-resolution, T1-weighted brain atlas provided by the Montreal Neurological Institute (MNI; the atlas is called MNI152) by using a mutual information algorithm with a 12-degrees-of-freedom transformation in the FSL Linear Image Registration Tool (FSL-FLIRT) (FMRIB, Oxford, UK: http://www.fmrib.ox.ac.uk/fsl/). ${ }^{10}$ Manual registration or adjustment was performed using Multiimage Analysis GUI (Mango, University of Texas Health Science Center: http://ric.uthscsa.edu/mango/ index.html) (Fig. 1). Appropriate image registration by FSL-FLIRT was visually assessed in all cases, with further manual adjustment required in 64 cases. Automatic registration by FSL-FLIRT was unsuccessful in 61 cases, and full manual registration was necessary for those cases.

After image registrations were completed, Gd-enhancing lesions were semiautomatically segmented using inhouse software developed on MATLAB (MathWorks). The Gd-enhancing lesion was first manually and coarsely segmented in 3 different orientations (axial, coronal, and sagittal), followed by a threshold-based further segmentation step. For each lesion, a 3D volume of interest was created, with lesion voxels assigned a value of 1 and nonlesion voxels a value of 0 . When multiple lesions were present, each one was analyzed separately. Then, lesions on the left side were flipped so that all lesions were located on the right side of the brain. This procedure was introduced to increase the number of overlapping samples and therefore the statistical power of the study, ${ }^{15}$ under the assumption that meningiomas evenly distribute at both sides of the brain. Finally, the volumes of interest were averaged to create a probability or heat map for the frequency of lesion occurrence. A 3D rendering of the frequency map was created using Mango software and superimposed on the surface rendering of brain extracted from the MNI152 (Fig. 1).

\section{Results \\ Validation by Comparison With Previous Publications}

A total of 260 lesions in 248 patients were included in the analysis. One hundred fourteen patients required surgical intervention to relieve neurological symptoms, whereas 134 patients were put under observation. Lesion locations were equally distributed between both hemispheres, and 13 lesions were categorized as multiple lesions. Table 2 summarizes lesion distribution based on anatomical locations compared with previous reports. The most common locations affected by these lesions were as follows: cranial convexity, parasagittal sinus, and parasel- 


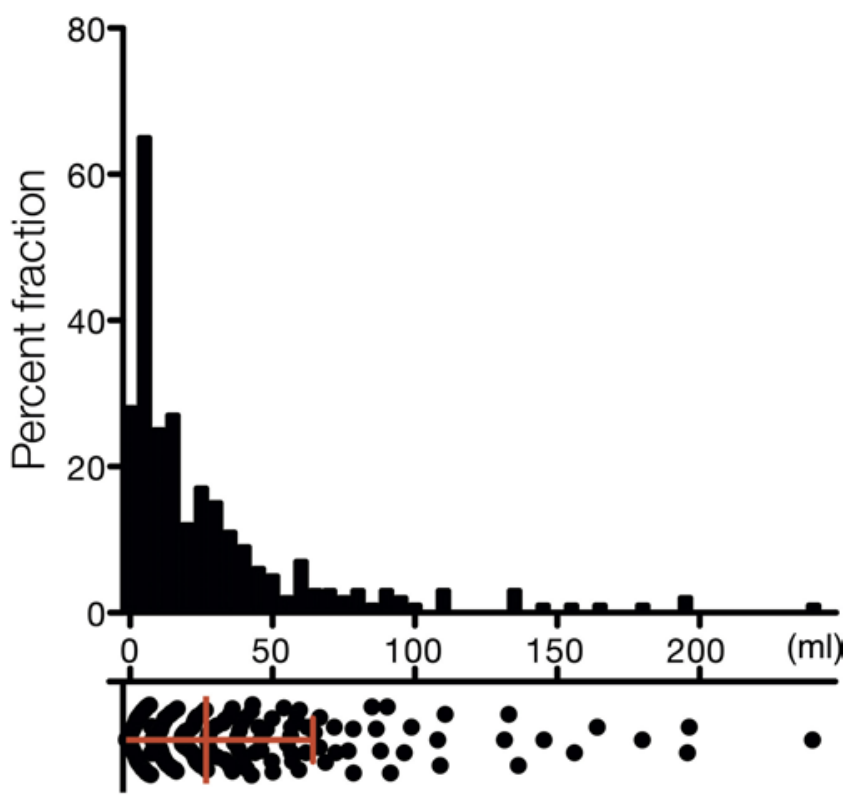

Tumor volume after MNI152 registration

FIG. 2. Graph showing tumor volume distribution after image registration. After each individual brain image was de-formed and registered on the MNI152, images were standardized and lesion sizes were calculated. The mean and median volumes were $28.1 \mathrm{ml}$ and $13.6 \mathrm{ml}$, respectively. Volume distribution did not fit a gaussian distribution $(p<0.0001$; D'Agostino and Pearson omnibus normality test).

lar or cavernous sinus. This tendency was in accordance with the Japan Brain Tumor Registry (JBTR) data from 2001 to 2004, the national epidemiologically accurate brain tumor database. ${ }^{5}$ Chi-square tests revealed that the current dataset and the JBTR did not differ in lesion location distribution $(p=0.83)$. These locations were also in line with the UCSF meningioma registration data, which showed that the cranial convexity and parasellar or cavernous sinus regions were the preferred locations where meningiomas occur. ${ }^{11}$ These observations suggest that the current data set corresponds to a homogeneously collected cohort of meningioma patients, and therefore allows further analysis under the assumption that selection bias is minimal.

\section{Voxel-Based Lesion Mapping of Meningiomas}

After each individual brain image was de-formed and registered onto MNI152 space, lesion sizes were calculated. The mean and median lesion volumes were $28.1 \mathrm{ml}$ and $13.6 \mathrm{ml}$, respectively. The lesion volume did not fit a gaussian distribution ( $\mathrm{p}<0.0001$; D'Agostino and Pearson omnibus normality test, Fig. 2). The frequency map of all the lesions is presented in Fig. 3, Video 1, and Supplementary Figs. 1-3.

VIDEO 1. A 3D rendering of the voxel-based lesion frequency map. Copyright Manabu Kinoshita. Published with permission. Click here to view.

The 3D lesion frequency map clearly showed that skull base structures such as parasellar, sphenoid wing, and petroclival regions were commonly affected by the tumor. With regard to parasagittal meningiomas, the frequency

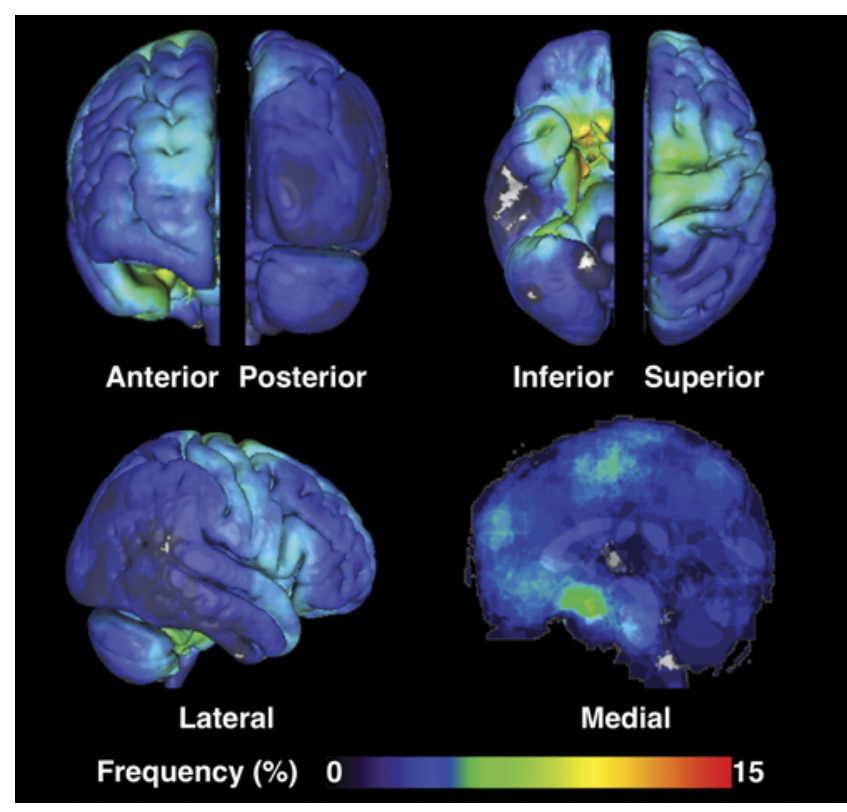

FIG. 3. A 3D rendering of the voxel-based lesion frequency map; 3D lesion frequency map clearly showing that skull base structures such as parasellar, sphenoid wing, and petroclival regions were commonly affected by the tumor. The middle one-third of the superior sagittal sinus was most commonly affected by parasagittal tumors. Noticeable lesion accumulation was observed around the leptomeninges covering the central sulcus and the sylvian fissure, with very few lesions observed at the frontal, parietal, and occipital convexities.

map revealed that the middle one-third of the superior sagittal sinus was substantially more commonly affected by the lesion than the anterior or posterior one-third. As for convexity meningiomas, lesion accumulation was mostly around the leptomeninges covering the central sulcus and the sylvian fissure. Very few lesions were observed at the frontal, parietal, and occipital convexities. When the frequency map was reconstructed and augmented by lesion size (Supplementary Fig. 4), the lesions seated at preferred locations tended to be larger. On the other hand, when the frequency map was regrouped according to treatment status (Supplementary Fig. 5), it was shown that lesions around the sylvian fissure and petrous apex were more likely to be treated nonoperatively, whereas midline lesions were likely to be treated operatively.

\section{Discussion}

The distribution of meningioma lesion locations has long been of clinical interest. The locations where meningioma occurs largely determine the patient's neurological symptoms, the extent of tumor removal, and the associated surgical risks and morbidity.1,17,20,23 Several reports argue that lesion locations may predict the speed of tumor growth. ${ }^{9,11}$ For example, it has been suggested that skull base lesions grow more slowly than those located in nonskull base regions. ${ }^{9}$

Due to their clinical relevance, lesion locations of meningiomas were thoroughly investigated by Harvey Cushing in the 1930s. ${ }^{718} \mathrm{He}$ described a series of locations where, from a neurosurgical point of view, meningiomas 


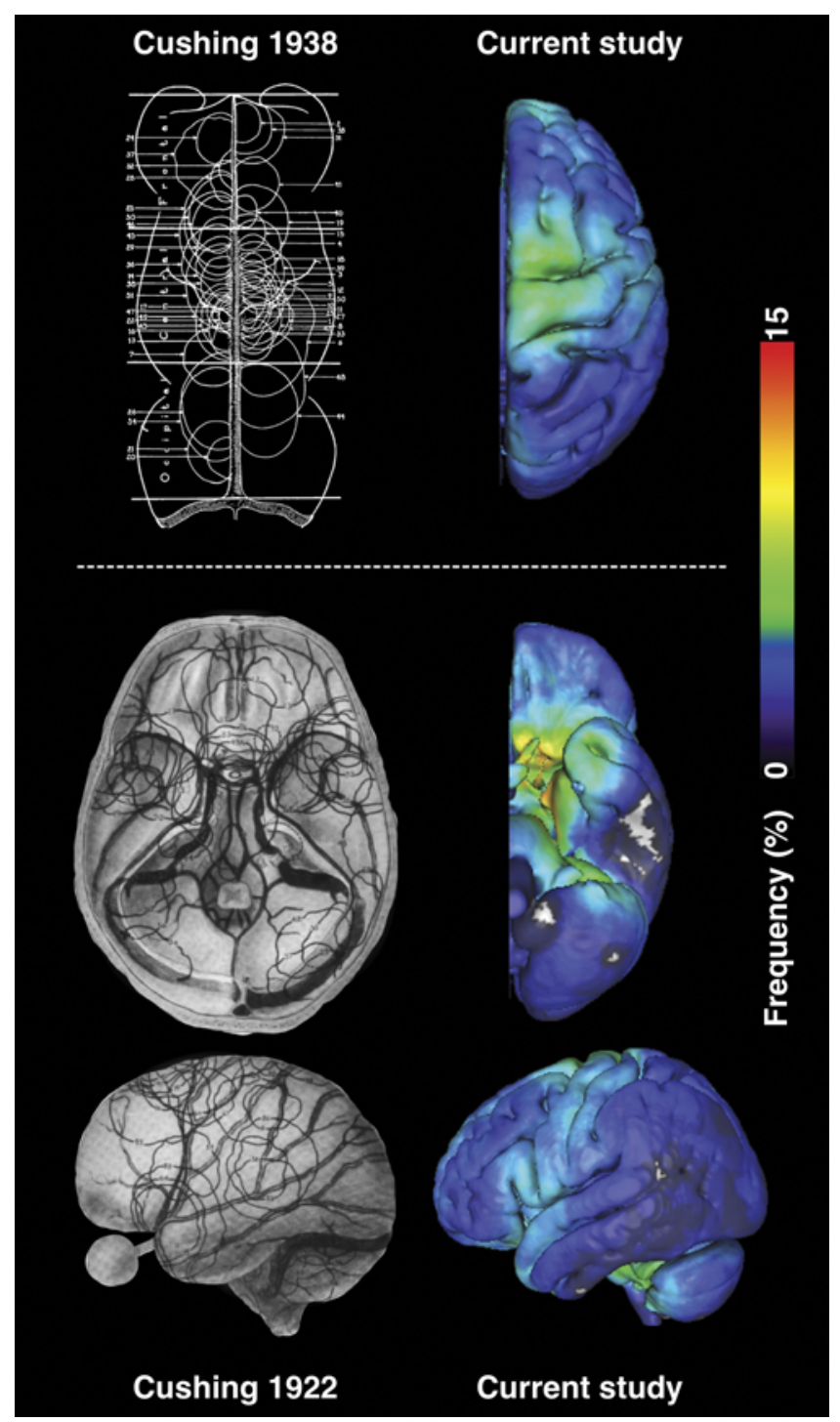

FIG. 4. Comparison of meningioma location preferences as documented by Harvey Cushing and in the current study. Both illustrations are in good agreement, showing that parasagittal meningiomas occur around the middle one-third of the superior sagittal sinus and that convexity meningiomas preferentially occur along the sylvian fissure, with very few located at the parietal convexity. The upper left image is from Harvey Cushing and Louise Eisenhardt's book Meningiomas, Their Classification, Regional Behaviour, Life History, and Surgical End Results (Springfield, IL: Charles C Thomas, 1938; public domain), and the 2 lower left images are from Harvey Cushing's paper "The meningiomas (dural endotheliomas): their source, and favoured seats of origin" (Brain 45:282-316, 1922; public domain).

were more common. Aiming to identify which tumors could be totally resected by sacrificing the superior sagittal sinus itself, he documented that meningiomas preferentially arise along the superior sagittal sinus. In particular, it was shown that the middle one-third of the superior sagittal sinus was the most commonly affected region. Our study agrees with these findings suggested in the 1930s, which had not been validated with current, more objective methodologies. The observation is also true for convexity meningiomas. The current study was able to identify that meningiomas tend to occur along the sylvian fissure and rarely reside at the parietal convexity (Figs. 3 and 4), as per Cushing's attempt to map convexity meningiomas to an atlas. The current study also revealed that meningiomas commonly appear in skull base regions.

The reason for location preferences in meningioma occurrence is unknown. One notable pattern that should be mentioned is that high-risk regions, as seen in Fig. 3, usually have thick and tough arachnoid membranes and relatively large CSF cisterns. ${ }^{21}$ It could be speculated that anatomical locations with high proportions of arachnoid cells are more frequently prone to harbor meningeal neoplasms. From a molecular biology point of view, several genomewide analyses have confirmed that meningiomas can be genetically classified as neurofibromatosis Type 2 (NF2) mutant and non-NF2 mutant, ${ }^{2-4,16}$ and that non-NF2 mutant meningiomas present oncogenic mutations in $A K T 1$ and $S M O{ }^{2,4}$ More interestingly, NF2 mutant meningiomas are reported to occur in hemispheric regions of the brain, whereas non-NF2 mutants concentrate in the midline of the cranium. ${ }^{4}$ These observations suggest that meningiomas in different locations may be biologically distinct. Combining voxel-based lesion mapping approaches, like the one used in the present study, with genetic information may provide more detailed information about the biology and pathology of meningiomas.

Several limitations should be addressed. First, the study's retrospective nature and the quality of the analyzed cohort may be criticized. Because so-called brain checkups are becoming more common in Japan, meningiomas are being identified more frequently in symptom-free patients..$^{8,9,13}$ However, more than half of the patients analyzed in the study were untreated (Table 1), which ensures that the current cohort is not biased in terms of tumor locations more frequently associated with neurological symptoms. The distribution of lesion locations in the current cohort did not differ from those in the literature. ${ }^{5,11}$ Another limitation is that the lesions were intentionally concentrated on one side of the brain. Although this method was previously used in a stroke study, the study design is based on the assumption that lesions are distributed equally in both hemispheres. ${ }^{15} \mathrm{~A}$ further large-scale study might be necessary to confirm our findings. And finally, the current study relied heavily on nonpathologically confirmed meningiomas, because nearly half of the patients did not undergo surgery. However, it is necessary to include these cases for analysis because incidentally discovered nonsymptomatic meningiomas are as important as those that manifest neurological deficits and require surgical intervention.

\section{Conclusions}

A voxel-based lesion mapping approach was used to quantitatively determine location preferences of meningiomas. It was shown that this neoplasm appears more commonly in the middle third of the superior sagittal sinus, the perisylvian convexity, and the skull base. These observations, which are in line with previous descriptive analyses, justify further use of the voxel-based lesion mapping technique to help understand the biological nature of this disease. 


\section{Acknowledgments}

This work was supported by the Japan Society for the Promotion of Science (JSPS) KAKENHI Grant No. 25462256 (https:// www.jsps.go.jp/english/index.html); Osaka Medical Research Foundation for Intractable Diseases (http://www.nanbyo.or.jp/ zaidan/info2013.html); Terumo Foundation for Life Sciences and Arts (https://www.terumozaidan.or.jp); The Uehara Memorial Foundation (http://www.ueharazaidan.or.jp); and Japan Brain Foundation (http://jbf.or.jp//).

\section{References}

1. Adachi K, Kawase T, Yoshida K, Yazaki T, Onozuka S: ABC Surgical Risk Scale for skull base meningioma: a new scoring system for predicting the extent of tumor removal and neurological outcome. Clinical article. J Neurosurg 111:1053-1061, 2009

2. Brastianos PK, Horowitz PM, Santagata S, Jones RT, McKenna A, Getz G, et al: Genomic sequencing of meningiomas identifies oncogenic SMO and AKT1 mutations. Nat Genet 45:285-289, 2013

3. Choy W, Kim W, Nagasawa D, Stramotas S, Yew A, Gopen $\mathrm{Q}$, et al: The molecular genetics and tumor pathogenesis of meningiomas and the future directions of meningioma treatments. Neurosurg Focus 30(5):E6, 2011

4. Clark VE, Erson-Omay EZ, Serin A, Yin J, Cotney J, Ozduman K, et al: Genomic analysis of non-NF2 meningiomas reveals mutations in TRAF7, KLF4, AKT1, and SMO. Science 339: 1077-1080, 2013

5. Committee of Brain Tumor Registry of Japan: Report of brain tumor registry of Japan (2001-2004). Neurol Med Chir (Tokyo) 54 (Suppl 1):1-102, 2014

6. Cushing H: The meningiomas (dural endotheliomas): their source, and favoured seats of origin. Brain 45:282-316, 1922

7. Cushing H, Eisenhardt L: Meningiomas, Their Classification, Regional Behaviour, Life History, and Surgical End Results. Springfield, IL: Charles C Thomas, 1938

8. Hashiba T, Hashimoto N, Izumoto S, Suzuki T, Kagawa N, Maruno M, et al: Serial volumetric assessment of the natural history and growth pattern of incidentally discovered meningiomas. J Neurosurg 110:675-684, 2009

9. Hashimoto N, Rabo CS, Okita Y, Kinoshita M, Kagawa N, Fujimoto Y, et al: Slower growth of skull base meningiomas compared with non-skull base meningiomas based on volumetric and biological studies. J Neurosurg 116:574-580, 2012

10. Jenkinson M, Beckmann CF, Behrens TEJ, Woolrich MW, Smith SM: FSL. Neuroimage 62:782-790, 2012

11. Kane AJ, Sughrue ME, Rutkowski MJ, Shangari G, Fang $\mathrm{S}, \mathrm{McDermott} \mathrm{MW}$, et al: Anatomic location is a risk factor for atypical and malignant meningiomas. Cancer 117:12721278,2011

12. Kinoshita M, Sasayama T, Narita Y, Yamashita F, Kawaguchi A, Chiba Y, et al: Different spatial distribution between germinal center B and non-germinal center B primary central nervous system lymphoma revealed by magnetic resonance group analysis. Neuro Oncol 16:728-734, 2014

13. Kuratsu J, Kochi M, Ushio Y: Incidence and clinical features of asymptomatic meningiomas. J Neurosurg 92:766-770, 2000

14. Onizuka M, Suyama K, Shibayama A, Hiura T, Horie N, Miyazaki H: Asymptomatic brain tumor detected at brain check-up. Neurol Med Chir (Tokyo) 41:431-435, 2001

15. Phan TG, Donnan GA, Wright PM, Reutens DC: A digital map of middle cerebral artery infarcts associated with middle cerebral artery trunk and branch occlusion. Stroke 36:986-991, 2005

16. Reuss DE, Piro RM, Jones DTW, Simon M, Ketter R, Kool $M$, et al: Secretory meningiomas are defined by combined KLF4 K409Q and TRAF7 mutations. Acta Neuropathol 125:351-358, 2013

17. Scheitzach J, Schebesch KM, Brawanski A, Proescholdt MA: Skull base meningiomas: neurological outcome after microsurgical resection. J Neurooncol 116:381-386, 2014

18. Shrivastava RK, Segal S, Camins MB, Sen C, Post KD: Harvey Cushing's Meningiomas text and the historical origin of resectability criteria for the anterior one third of the superior sagittal sinus. J Neurosurg 99:787-791, 2003

19. Takano K, Kinoshita M, Takagaki M, Sakai M, Tateishi S, Achiha T, et al: Different spatial distributions of brain metastases from lung cancer by histological subtype and mutation status of epidermal growth factor receptor. Neuro Oncol 18:716-724, 2016

20. van Alkemade H, de Leau M, Dieleman EMT, Kardaun JWPF, van Os R, Vandertop WP, et al: Impaired survival and long-term neurological problems in benign meningioma. Neuro Oncol 14:658-666, 2012

21. Yaşargil MG: Microsurgical Anatomy of the Basal Cisterns and Vessels of the Brain. Stuttgart: Thieme, 1984

22. Yoneoka Y, Fujii Y, Tanaka R: Growth of incidental meningiomas. Acta Neurochir (Wien) 142:507-511, 2000

23. Zentner J, Meyer B, Vieweg U, Herberhold C, Schramm J: Petroclival meningiomas: is radical resection always the best option? J Neurol Neurosurg Psychiatry 62:341-345, 1997

\section{Disclosures}

The authors report no conflict of interest concerning the materials or methods used in this study or the findings specified in this paper.

\section{Author Contributions}

Conception and design: Kinoshita, Hirayama, Hashimoto, Yoshimine. Acquisition of data: all authors. Analysis and interpretation of data: Kinoshita, Hirayama, Arita, Kishima, Hashimoto, Fujimoto, Yoshimine. Drafting the article: Kinoshita. Critically revising the article: Kinoshita, Yoshimine. Reviewed submitted version of manuscript: Kinoshita. Approved the final version of the manuscript on behalf of all authors: Kinoshita. Statistical analysis: Kinoshita. Administrative/technical/material support: Kinoshita. Study supervision: Kinoshita.

\section{Supplemental Information \\ Videos}

Video 1. https://vimeo.com/215514029.

Online-Only Content

Supplemental material is available with the online version of the article.

Supplementary Figures. https://thejns.org/doi/suppl/10.3171/ 2017.3.JNS17169.

\section{Correspondence}

Manabu Kinoshita, Department of Neurosurgery, Osaka International Cancer Institute, 3-1-69 Otemae, Chuo-ku, Osaka 541-8567, Japan.email: mail@manabukinoshita.com. 